\title{
Optimization of Unit Commitment Problem Using Genetic Algorithm
}

\author{
Aniket Agarwal, Gautam Buddha University, India \\ Kirti Pal, Gautam Buddha University, India
}

\begin{abstract}
The main objective of the paper is to minimize the use of conventional generators and optimize the fuel cost. To minimize the use of conventional generators, solar thermal power plant (STPP) is proposed in this paper. An approach for optimal location of STPP is also proposed in this paper. To minimize the fuel cost, firstly unit commitment (UC) is applied in conventional generators. Then genetic algorithm (GA) is used to optimize the fuel cost of committed generators. The suggested method is tested on an IEEE 14 bus test system for $24 \mathrm{hr}$. schedule with variable load. The effectiveness of the proposed methodology is illustrated in three cases. Case 1 is used to identify the STPP location to reduce the fuel cost of conventional generator. In Case 2, unit-commitment is applied to save considerable fuel input and cost. In order to optimize the committed fuel cost, a genetic algorithm is applied in Case 3 .
\end{abstract}

\section{KEYWORDS}

Alternative Current Optimal Power Flow, Genetic Algorithm, IEEE 14 Bus System, MATPOWER, Power System Constraints, Renewable Sources, Solar Thermal Power Plant, Unit Commitment

\section{INTRODUCTION}

Electrical power systems are designed and operated to meet the continuous variation of load demand. In power system minimization of operation cost is very important. The most important problem with electric power generation scheduling is unit commitment. Unit Commitment means coordinating short-term unit generation to meet load demand forecasting. The problem of unit commitment is a complex problem of optimization. It has a variable integer as well as a constant variable (Kumar et al., 2014). The Unit Commitment (UC) function in the power system refers to the question of optimization to evaluate the on/off states of generating units that reduce operating costs for a given time horizon (Zhao et al., 2006). The committed units must meet the system's forecast demand and spinning reserve requirement at the minimum operating cost, subject to a wide set of operating restrictions.

In practice as well as in state-of-the-art research on deterministic UC models, the underlying security assumptions (i.e., contingency) network, and operational constraints for alternating current (AC) power systems have varied the most significantly described by (Stott \& Alsac, (2012). Garver., (1962) improves the foundational formulation of UC. Several influential approaches have concentrated on mixed-integer linear programming (MILP) with more close convex null representations of non- 
network thermal unit constraints operation described in several research papers (Carrion \& Arroyo, 2011); (Ostrowski et al., 2012); (Morales-Espana et al., 2013); (Jabr, 2012). Unit Commitment in linear (DC) network with losses are formulated by (Morales et al., 2009) and (Wu \& Shahidehpour, 2010) and without is formulated by (Feizollahi et al., 2015).

Padhy, (2004) explain how the resulting commitment schedules neglect the constraints of reactive power dispatch and AC power flow, which must subsequently be compensated for via corrective and generally ad-hoc processes. The resultant interaction schedules ignore the constraints of reactive power dispatch and ac power flow, which must consequently be balanced by corrective and typically ad-hoc processes explained by (Bhardwaj et al., 2012). It is the energy control center's most critical feature that specifies the on / off status as well as the actual power output of the generator. Parashar \& Swankar, (2013), proposes a methodology to reduce device running costs over the planning cycle subject to various physical operation and computation constraints. Columbus \& Simon, (2012), explain unit Commitment (UC) as a nonlinear mixed integer optimization problem. Power system engineers also need to understand the economic decision-making tool for to accomplish the economic goal (Galli, 2019).

With rapid development of the world economy and the exhaustion of the fossil fuels, renewable energy sources (RES) has received significant attention in the current society. Several authors (Wei et al., 2013); (Danwen et al., 2016); (Shin et al., 2017) explain the characteristics of renewable energy sources as a non-pollution and inexhaustible. The wind energy has become one of the renewable sources of electricity generation. Author Hirpara \& Sharma, (2020), utilizes the Fokker-Planck method, a mathematical stochastic method, to analyse the noise-influenced wind turbine-generator system. The solar energy is another most popular example of renewable sources of energy. To make a full utilization of the output power of a PV solar cell operating at the maximum power point (MPP) is described in detail by Yatimi, H. (2018). Some researcher work on a practical case study such as (Mohamed et al., 2017) for 20 MW Egyptian PV solar power plant with battery backup system. Singh et al. (2019) evaluate the performance of a $4 \mathrm{~kW}$ Isolated Solar Powered Lab with IoT Energy Management System. Some applications based on solar energy is also discussed by (Singh et al. 2018) and (Salima et al. 2018).

Various emerging concepts influence logistics management (Baporikar 2020), particle swarm optimization, fuzzy logic controller (Ramadan \& Altamimi 2017) and genetic algorithm etc are general purpose stochastic and parallel search tool that can be used as a technique of optimization to obtain near-global optimal solutions to the problem. The Genetic algorithm is inspired by the ideas of the natural selection and survival of the fittest in biology and evolution. Lambora et al. (2019), present review on genetic algorithm application. Many authors such as (Meshram et al.,2016) and (Xua, et. al. 2018) explain the Genetic algorithm applications in different areas.

The main objective of the paper is to minimize the fuel cost of conventional generators with the inclusion of renewable energy sources and optimization technique. The paper is organized in different sections. In background section unit commitment and its importance and UC with renewable power system is discussed. In second section problem formulation and genetic algorithm optimization techniques are described in detail. In third section numerical results are explained in three different cases to show the effectiveness of the proposed methodology. Finally conclude with the findings of proposed methodology.

\section{BACKGROUND}

\section{Unit Commitment and its Importance}

Economic operation results in increasing operational efficiencies, thereby minimizing costs per kilowatt-hour. Total power system load varies at any time, generally higher during the daytime and early evening when industrial loads are heavy, lights are on, and so on, and lower in the late night and 
early morning when the majority of the population sleeps. The option to turn ON enough units and leave them online is therefore not viable due to the costs involved so that the variable load demand is met at all times. This occasionally causes some of the units to operate close to their minimum capacity, leading to lower system efficiency and increased economics. Therefore, in order to optimize device operation, units must be shut down as the load goes down and must be brought online as it goes up again (Stott \& Alsac, (2012).

Electrical utilities must schedule their generation in advance to meet this varying demand, as to which generators are to be started and when to synchronize them into the network and the sequence where working systems have to be shut down. The decision-making process is well-known as " Unit Commitment'. The word ' commit ' refers to making a turn 'ON' a unit. Therefore, the Unit Commitment issue is to plan the ON and OFF hours of the generating units with the overall minimum cost while maintaining the operating restrictions of the unit, such as peak up / down periods, ramp rate limits, maximum and minimum power generation limits. The main component of the costs incurred in generation is the cost of fuel supply per hour for all generators, while maintenance costs only contribute to a small extent. This assessment of the fuel costs is more relevant for thermal and nuclear power plants, which is not the case for hydro stations where the energy is derived from the storage of water in dams built for irrigation purposes and is apparently free. Savings in fuel costs can be achieved by proper load allocation among the engaged units.

\section{UC with Renewable Resources in Power System}

With the prolonged and excessive use it has become clear that supplies of fossil fuel are increasingly depleting and that the age of fossil fuel is slowly coming to a close. This is particularly true of coal, oil, and natural gas resources. The large-scale use of fossil fuels has caused detrimental environmental effects over the years. The result of this is the movement towards global warming which is of great concern for the future of human life. The pursuit of alternative sources, the situation has caused the whole world to meet the energy needs.

Renewable energy sources such as solar, wind, tidal and geothermal, were the alternatives that attracted the attention. It is a success to extract energy from the solar and wind to a considerable level. The research focuses on the use of solar thermal energy for electricity generation here. For model a solar integrated power system near a realistic scenario, a solar thermal power plant is installed into the existing power grid.

\section{PROBLEM FORMULATION}

Electricity demand is higher in the day time and lower in the late night and early morning. The cyclical demand includes hourly preparation by utility companies for power generation. The issue is to decide which of the units to be turn on, and then to assess the units for an optimal Economic dispatch schedule for a set of Unit Committed generating systems.

\section{Objective Function}

The objective is that the fuel cost of all committed generators is to be minimized in (1). The simplified cost function of every generator can be represented as a quadratic function as given in (2).

$$
F\left(P_{g i}\right)=\min \sum_{i=1}^{N G} F_{i}\left(P_{g i}\right)
$$

subject to: 


$$
F_{i}\left(P_{g i}\right)=a_{i} P g_{i}^{2}+b_{i} P g_{i}+c_{i}
$$

Here:

$a_{i}, b_{i}$ and $c_{i}$ are the known coefficients for $i^{t h}$ unit

$N G$ : number of generators

$P g_{i}:$ real power generation of $i^{\text {th }}$ plant

$F_{i}\left(P_{g i}\right):$ the total cost of generation

\section{Equality and Inequality Constraints}

\section{Active Power Balance Equation}

For power balance, an equality constraint should be acquiescent. The total generated power should be the same as total load demand plus the total transmission line loss:

$$
\sum_{i=1}^{N G} P_{g i}, P_{D}, P_{l o s s}
$$

where $P_{D}$ is the total load demand and $P_{\text {loss }}$ is the total line loss.

\section{Minimum and Maximum Power Limits}

Generation output of each generator should be lie between maximum and minimum limits. The compatible inequality constraints for each generator are:

$$
p_{i}^{\min } \leq p_{i} \leq p_{i}^{\max }(i=1,2, \ldots, N G)
$$

where $P_{g i}^{\min }$ is the lower permissible limit of real power generation, $P_{g i}^{\max }$ is the upper permissible limit of real power generation.

\section{GENETIC ALGORITHM}

Genetic algorithms are known as the heuristics of global searches. Genetic algorithm is a special class of Evolutionary Algorithms (EA), using evolutionary biology-inspired techniques such as inheritance, selection, crossover, and mutation. A Genetic Algorithm (GA) is a computational search technique used to find exact or approximate solutions to search and optimization problems. The Genetic algorithm is based on principle that "The candidates, which can survive, will live, others would die". This principle is used to finding fitness value of the process for solving optimization problems. It is a method that is based on natural selection, the process that drives biological evolution.

When we have the genetic representation and the fitness function prescribe, GA initializes a population of solutions randomly, and then enhances the treatment of mutation, inversion, crossover and selection operators for repeated use. For binary, solutions are usually represented as 0s and 1s strings but other encoding is also possible. Genetic algorithms are implemented in a computer simulation in which a population of abstract representations (called chromosomes or genotypes of 
the genome) of candidate solutions (called organisms, individuals, or phenotypes) to an optimization problem evolves towards better solutions. If due to a maximum number of generations, the algorithm has ended, a suitable solution may or may not have been found. The evolution typically begins from a population of individuals that are randomly generated and happens in generations. Multiple individuals in each generation are stochastically selected from the current population based on their fitness. The fitness of each individual in the population is evaluated and changed (recombined and likely mutated at random) to create a new population. Commonly, the algorithm ends when either a maximum number of generations have been made, or a sufficient fitness level for the population has been reached. For the next iteration of the algorithm the new population is then used. The flow chart of genetic algorithm is shown in Figure 1.

\section{Representation}

In GA, the variables of design or features characterizing an entity are expressed in an ordered list called a string. Genetic algorithms are extracted from a biological method analysis. Evolution happens in biological system on organic devices used to represent the form of living beings. Every design variable

Figure 1. Flow chart of Genetic Algorithm

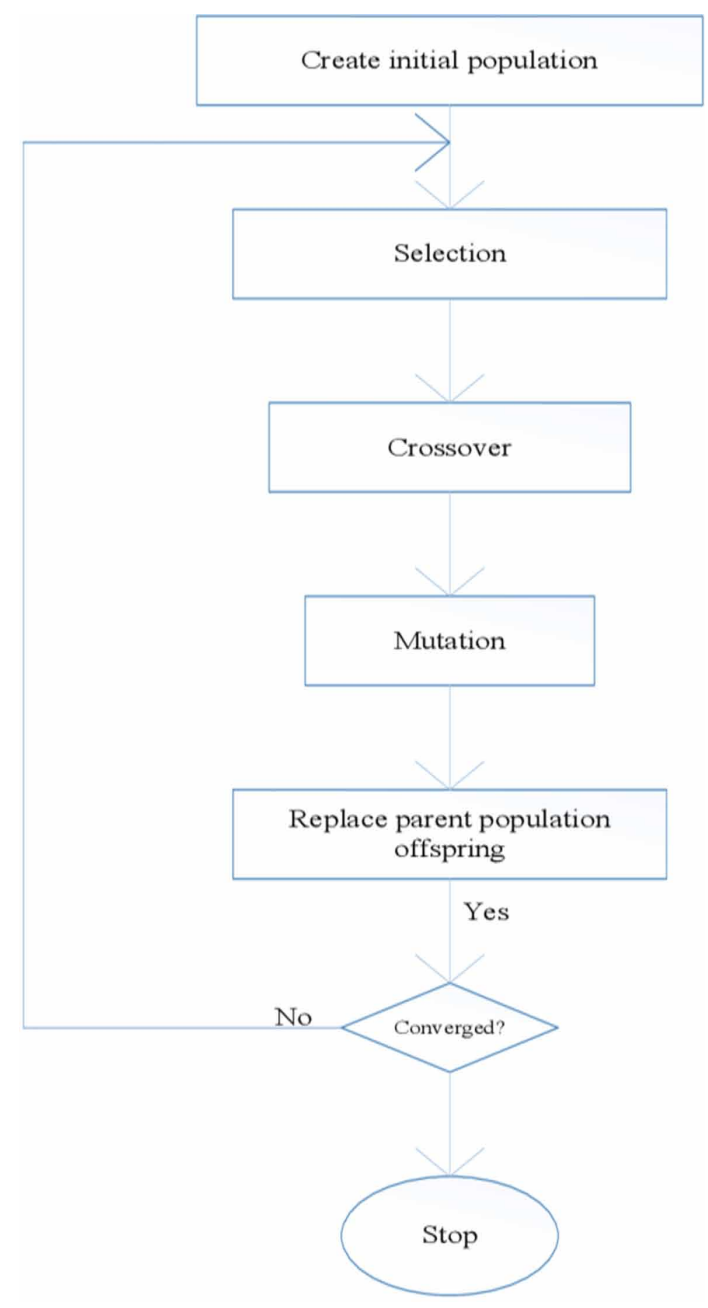


is the same as a gene and the gene string corresponds to a chromosome. Chromosomes are composed of discrete units called chromosome. Natural selection is the interface between chromosomes and their decoded structures results. Such biological apparatuses are known as chromosomes. A human thing is just a decoded chromosomal fabrication.

\section{Encoding}

This includes a mapping process between chromosomal space and solution space. This mapping is called encoding. Typically, a chromosome in solution space corresponds to a single solution $\mathrm{x}$. The encoding starts with the application of a genetic algorithm to a query. In fact, GA is working on the encoding of an issue not the problem itself. The encoding specifies a mapping which translates a potential solution to the problem into a structure which contains a set of relevant decision variables.

\section{Decoding}

Decoded feature values are used to measure problem characteristics such as fitness values, violation of constraints, objective function, and statistical system characteristics such as standard variance deviation and convergence rate. Crossover steps, selection, mutation etc. are replicated until some state of termination is reached. Decoding is the method of translating the chromosomal binary structure into decimal versions of the characteristic values. The procedure is usually performed after the entire chromosome has been decatenated to individual chromosomes. The equivalent decimal integer of binary string is obtained as $y^{j}=\sum_{i=1}^{l} 2^{i-1} b_{i}^{j}(\mathrm{j}=1,2, \ldots ., \mathrm{L})$; where, $y^{j}$ is the binary coded value of the string, $b_{i}^{j}$ is the $i^{\text {th }}$ binary digit of the $j^{\text {th }}$ string, 1 is the length of the string, $\mathrm{L}$ is the number of strings or population size.

\section{Initialization}

Initially several individual solutions are created randomly for forming an initial population. The solutions can sometimes be "seeded" in areas where optimum solutions are likely to be found. Traditionally, population is generated randomly that covers the whole range of possible solutions (the search space). The size of the population depends on the complexity of the case but usually involves several hundreds or thousands of potential solutions.

\section{Evaluation}

The evaluation function is a method for evaluating the fitness of each chromosome in the population and is very much geared towards application. This function is derived from the function of the target and used in the genetic process. The suitability of the solutions is determined from the initial problem-solving package. Here a function called fitness function for this appropriateness allocation. In the case of routines for command interpreters, the fitness is the value to be optimized for the objective purpose. In order to achieve a constrained problem, penalty functions can also be used in the objective function. Since Genetic Algorithms are heading in the direction of developing the most suited chromosomes and the output is highly sensitive to fitness.

\section{Fitness Function}

The fitness value $f(x)$ is derived from the aim function and is used in successive genetic operations. Using some suitable transformations, minimization problems are typically moved to problems of maximization. The Genetic algorithm is based on the theory of Darwin that "the candidates who can survive will live, others will die". This theory is used for finding process fitness value to solve problems with maximization. The maximization problem fitness function can be used exactly the same as the objective function $\mathrm{F}(\mathrm{X})$. The fitness function for the maximization problem is: $\mathrm{f}(\mathrm{x})=$ $\mathrm{F}(\mathrm{X})$. The fitness function is an analogous maximization problem chosen such that the optimum point 
Arrears is stationary for minimization problems. The following exercise function is mostly used in problems of minimization:

$$
F(X)=\frac{1}{1+f(x)}
$$

Here $\mathrm{f}(\mathrm{x})$ is fitness function and $\mathrm{F}(\mathrm{X})$ is objective function.

\section{Selection}

Individual solutions are selected by a fitness-based process in which fitter solutions (as calculated by a fitness function) are usually more likely to be selected. Some selection methods rate each solution's fitness and ideally select the best extrication. Other methods only score a random population sample, as this procedure can take a great deal of time. A proportion of the current population is chosen to raise a new generation during each continuous generation. This helps to preserve the diversity of the population, preventing premature convergence on beggarly solutions. Roulette wheel selection and tournament selection are common and well-studied methods of selection. Most of the functions are stochastic and built to choose a small portion of less-fit solutions.

\section{Reproduction}

The further move is to produce a second-generation population of solutions from those selected by genetic operators: crossover (also known as recombination) and/or mutation. By generating a "kid" solution using the above crossover and mutation methods, a new solution is produced that usually shares many of its "parents" characteristics. A pair of "parent" solutions is chosen for each new solution to be created for breeding from the pool previously chosen. New parent is chosen for each new infant, and the process continues until a new population of suitable size solutions is created. Although methods of reproduction focused on the use of two parents are more "inspired by biology," some work indicates that it is safer to use more than two "parents" for reproducing a good quality chromosome. This procedure would usually increase the average fitness for the population, as only the best species of the first generation are selected for reproduction, along with a small proportion of less suitable solutions, for reasons already stated above. Ultimately, these processes result in the next generation chromosome population which is distinct from the initial generation.

\section{Termination}

This generational cycle is replicated until a state of termination is attained. Specific ending conditions are as follows:

1. A solution which meets minimum requirements is found.

2. The number of generations achieved is set.

3. Assigned budget (time/money for calculation) met.

4. The fitness of the higher ranking solution approaches or has reached a plateau such that successive iterations no longer produce better results.

\section{Proposed Algorithm for GA}

1. Read data, namely cost coefficients, $a_{i}, b_{i}, c_{i}$ no. of iterations, length of string, population size, probability of crossover and mutations, power demand and $P_{\min }$ and $P_{\max }$.

2. Create the initial population randomly in the binary form. 
pop=round(rand(popsize, $\mathrm{Nt})$ );

$\mathrm{Nt}=$ total number of bits in a chromosome

3. Decode the string, or obtain the decimal integer from the binary string using Eq.5:

$$
y^{j}=\sum_{i=1}^{l} 2^{i-1} b_{i}^{j}(\mathrm{j}=1,2, \ldots \ldots, \mathrm{L})
$$

where, $y^{j}$ is the binary coded value of the string, $b_{i}^{j}$ is the $i^{\text {th }}$ binary digit of the $j^{\text {th }}$ string, 1 is the length of the string, $\mathrm{L}$ is the number of strings or population size.

4. Calculate the power in MW generated from the decoded population by using Eq.(6):

$$
p_{i}^{j}, p_{i}^{\min }+\frac{p_{i}^{\max }-p_{i}^{\min }}{2^{l}-1} y_{i}^{j}(\mathrm{i}=1,2, \ldots . \mathrm{NG}, \mathrm{j}=1,2, \ldots, \mathrm{l})
$$

where, $y_{i}^{j}$ is the binary coded value of the $i^{t h}$ substring.

5. Check $p_{i}^{j}$, if $p_{i}^{j}, p_{i}^{\max }$ then set $p_{i}^{j}=p_{i}^{\max }$, if $p_{i}^{j}, p_{i}^{\min }$, then set $p_{i}^{j}=p_{i}^{\min }$.

6. Find fitness if $\left(f_{i}, f_{\max }\right)$ then set $f_{\text {max }}, f_{i}$. If $\left(f_{i}, f_{\min }\right)$ then set $f_{\min }, f_{i}$.

7. Find population with maximum fitness and average fitness of the population.

8. Select the parents for crossover using stochastic remainder roulette wheel selection method.

9. Perform single point crossover for the selected parents.

10. Perform mutation

11. If the number of iterations reaches the maximum, then go to step 12. Otherwise, go to step 2.

12. The fitness that generates the minimum total generation cost is the solution of the problem.

\section{RESULT AND DISCUSSIONS}

The proposed methodology is implemented on standard IEEE 14bus test system. The results of simulation studies are illustrated in three cases.

\section{Case 1: Optimal Location of STPP}

The data of IEEE 14 bus system is taken from MATPOWER6 software. As we know IEEE 14 bus system consists of 5 conventional generators. Even when we run OPF program for an IEEE 14 bus system using MATPOWER6 the cost applied to consumer is very high. As it is well known that OPF is very different from ordinary power flow. Optimal power flow (OPF) is an extension to conventional ED problem; it determines minimal cost by optimal settings of different control variables in the system.

In order to minimize the conventional generator fuel cost STPP is integrated after some modification in MATPOWER6 IEEE 14 bus system data. STPP has to be added as an extra plant into the existing system. To identify the optimal location for integration of STPP, load at each bus is increased by $10 \%$ and then by $20 \%$ to observe the impact on consumer cost.

Table 1 show that under base case, with $10 \%$ increased load and with $20 \%$ increased load the consumer end cost is higher at bus number 13 and 14. To minimize the cost and to minimize the use of conventional generator two STPP with capacity of $10 \mathrm{MW}$ each is integrated at bus 13 and 14 . 
Table 1. Load impact on consumer cost

\begin{tabular}{|l|l|l|l|}
\hline \multirow{2}{*}{ Bus No: } & \multicolumn{1}{|c|}{ Base case (259 MW) } & \multicolumn{1}{c|}{$\begin{array}{c}\text { With 10\% increase in load } \\
(\mathbf{2 8 4 . 9} \mathbf{M W})\end{array}$} & $\begin{array}{c}\text { With 20\% increase in load (310.8 } \\
\text { MW) }\end{array}$ \\
\cline { 2 - 4 } & \multicolumn{1}{|c|}{ Cost(\$/hr) $\mathbf{~} / \mathbf{h r})$} & \multicolumn{1}{c|}{ Cost(\$/hr) } \\
\hline 1 & 36.724 & 36.925 & 37.061 \\
\hline 2 & 38.360 & 38.590 & 38.754 \\
\hline 3 & 40.575 & 40.789 & 40.970 \\
\hline 4 & 40.190 & 40.437 & 40.603 \\
\hline 5 & 39.661 & 39.917 & 40.068 \\
\hline 6 & 39.734 & 40.030 & 40.160 \\
\hline 7 & 40.172 & 40.394 & 40.570 \\
\hline 8 & 40.170 & 40.390 & 40.564 \\
\hline 9 & 40.166 & 40.380 & 40.564 \\
\hline 10 & 40.318 & 40.567 & 40.767 \\
\hline 11 & 40.155 & 40.438 & 40.618 \\
\hline 12 & 40.379 & 40.742 & 40.942 \\
\hline $\mathbf{1 3}$ & $\mathbf{4 0 . 5 7 5}$ & $\mathbf{4 0 . 9 4 4}$ & $\mathbf{4 1 . 1 6 8}$ \\
\hline $\mathbf{1 4}$ & $\mathbf{4 1 . 1 9 7}$ & $\mathbf{4 1 . 5 7 3}$ & $\mathbf{4 1 . 8 7 3}$ \\
\hline
\end{tabular}

\section{Case 2: Implementation of UC-ACOPF in Standard IEEE 14 Bus Test System With STPP}

Here UC- ACOPF is scheduled for 24-hour to show the effectiveness of proposed methodology. A load profile is developed for 24-hour in order to keep in mind that during office hours load is high while in morning, lunch time and late-night load is low. Figure 2 show the load profile for a scheduled day. Load variation is clearly visible in Figure 2. Hour 1 is started from mid night.

Figure 2. Load profile for 24 Hour

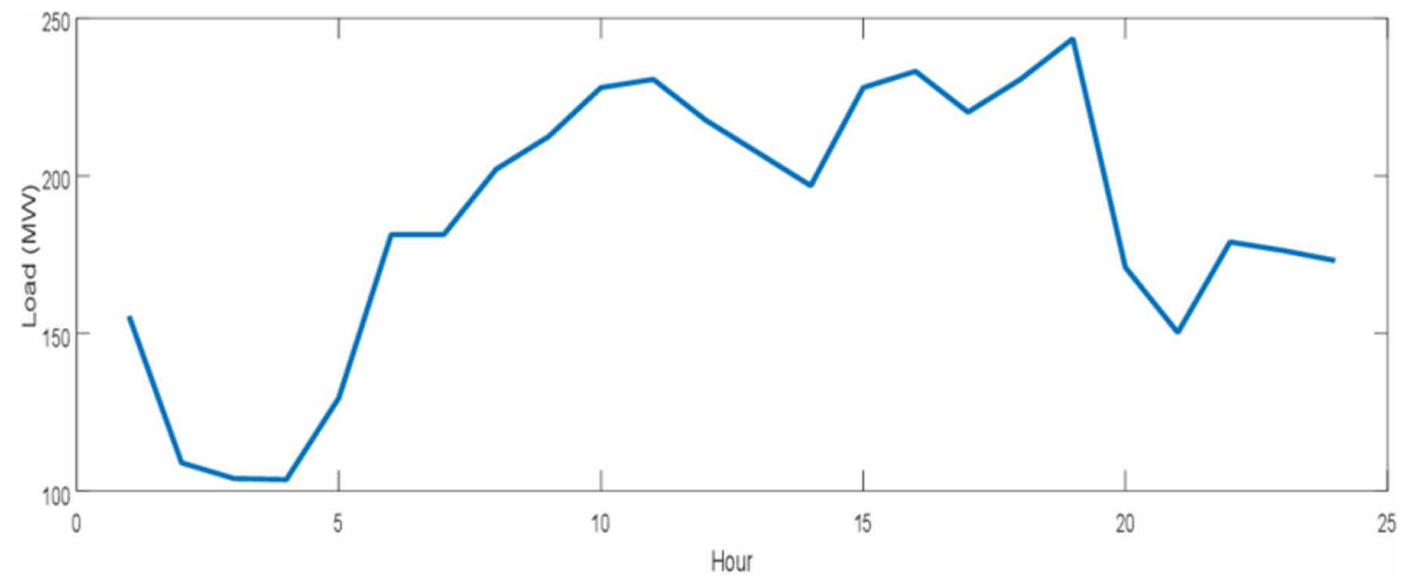


As the STPP can work in day time only the following schedule for generating units are assumed:

1. During 12:00 am to 09:00 am, only the conventional thermal units have to cater the load.

2. During 09:00 am to 6:00 pm conventional units and solar thermal unit caters the load.

3. During 6:00 pm to 11:00 pm again only conventional units caters the load.

It is clear from the time frame that during day time or office peak hours STPP will remain ON. During non-availability or low output periods of solar generation, load demand has to be catered by the other units reliably without violating any operational constraints. Thus, the choice of generating unit that is to be substituted by the modelled STPP is required to be judicious.

As the optimal location is identifies in case 1, add the STPP in bus no.13 and 14 of standard IEEE 14 bus test system. After modification (implementation of STPP) in IEEE 14 bus system Unit Commitment is scheduled for every hour of the day by using MATPOWER6 software.

\section{Algorithm for 24-hour UC With STPP Integration}

Step 1: During early hours and evenings, the STPP output is too low to run the unit and is taken as zero. However, during day time from 9:00am to 6:00pm the STPP output is taken constant of $10 \mathrm{MW}$ each. Therefore, total generation capacity of two STPP, $P_{g, S T P P}=20 \mathrm{MW}$.

Step2: Sum up the loads at all buses to obtain the total hourly demand.

Step 3: In order to commit the units to cater primarily to system demand it is assumed that all units are available for commitment over the entire UC planning period of 24 hours.

Step 4: The spinning reserve requirements of the system are considered to be $10 \%$ of the system load. This is added to what was determined in step 3 to find the total MW generation required to be committed.

Step 5: Since two of the generators is treated as STPP $\left(P_{g, S T P P}\right)$ the calculated hourly fixed power output of it is subtracted from the total MW generation requirement. Commitment schedule is determined every hour among only the remaining $(N G-1)$ generators to cater to this new net generation requirement. In this regard the modified or new net generation requirement is given as below:

$$
P_{g, \text { new }}=\left(P_{D}+\text { losses }+ \text { Spinning Reserve }\right)-P_{g, S T P P}
$$

Step 6: Run UC-ACOPF program for modified IEEE 14 bus system using MATPOWER6 software to determine the optimum generation cost for the hour's commitment.

Step 7: Repeat step 1 to step 6 for every hour.

Table 2 shows the UC schedule for 24 hours along with unit status, total generation and hourly generation cost in presence of STPP. During peak load hour such as for hour 10, 11, 15, 16, 18 and 19 total generation cost is considerably reduced while using STPP as compared with other load hours without STPP.

Table 3 shows the maximum voltage, minimum voltage, active and reactive loss for 24-hour UC schedule with STPP. The voltages at load buses in the system during 24-hour time period attained as high as 1.06 PU and as low as 1.012 PU.

\section{Case 3: Implementation of GA for Unit Committed Conventional Generators}

The main focus of the paper is to minimize the use of conventional generators with optimized fuel cost. 
Table 2. UC for IEEE 14 bus system with STPP

\begin{tabular}{|c|c|c|c|c|c|c|c|c|c|c|c|c|c|c|c|c|c|}
\hline \multirow{2}{*}{$\begin{array}{c}\text { Time } \\
\text { (hr) }\end{array}$} & \multirow{2}{*}{$\begin{array}{l}\text { Load } \\
\text { (MW) }\end{array}$} & \multicolumn{7}{|c|}{ Unit status } & \multicolumn{7}{|c|}{ Power output (MW) } & \multirow{2}{*}{$\begin{array}{l}\text { Total } \\
\text { Power } \\
\text { (MW) }\end{array}$} & \multirow{2}{*}{$\begin{array}{c}\text { Total } \\
\text { Cost } \\
(\$ / \mathbf{h r})\end{array}$} \\
\hline & & 1 & 2 & 3 & 6 & 8 & 13 & 14 & 1 & 2 & 3 & 6 & 8 & 13 & 14 & & \\
\hline 1 & 155.40 & 1 & 1 & 0 & 0 & 0 & 0 & 0 & 134.91 & 25.16 & 0 & 0 & 0 & 0 & 0 & 160.07 & 4142 \\
\hline 2 & 109.00 & 1 & 1 & 0 & 0 & 0 & 0 & 0 & 93.78 & 17.32 & 0 & 0 & 0 & 0 & 0 & 111.1 & 2675 \\
\hline 3 & 104.00 & 1 & 1 & 0 & 0 & 0 & 0 & 0 & 89.25 & 16.46 & 0 & 0 & 0 & 0 & 0 & 105.71 & 2524 \\
\hline 4 & 103.60 & 1 & 1 & 0 & 0 & 0 & 0 & 0 & 89.25 & 16.46 & 0 & 0 & 0 & 0 & 0 & 105.71 & 2524 \\
\hline 5 & 129.50 & 1 & 1 & 0 & 0 & 0 & 0 & 0 & 111.98 & 20.77 & 0 & 0 & 0 & 0 & 0 & 132.75 & 3302 \\
\hline 6 & 181.30 & 1 & 1 & 0 & 0 & 0 & 0 & 0 & 158.04 & 29.64 & 0 & 0 & 0 & 0 & 0 & 187.68 & 5047 \\
\hline 7 & 181.30 & 1 & 1 & 0 & 0 & 0 & 0 & 0 & 158.04 & 29.64 & 0 & 0 & 0 & 0 & 0 & 187.68 & 5047 \\
\hline 8 & 202.02 & 1 & 1 & 0 & 0 & 0 & 0 & 0 & 176.69 & 33.29 & 0 & 0 & 0 & 0 & 0 & 209.98 & 5819 \\
\hline 9 & 212.38 & 1 & 1 & 0 & 0 & 0 & 1 & 1 & 167.96 & 31.62 & 0 & 0 & 0 & 10 & 10 & 219.58 & 5455 \\
\hline 10 & 227.92 & 1 & 1 & 0 & 0 & 0 & 1 & 1 & 181.97 & 34.37 & 0 & 0 & 0 & 10 & 10 & 236.34 & 6046 \\
\hline 11 & 230.51 & 1 & 1 & 0 & 0 & 0 & 1 & 1 & 184.30 & 34.83 & 0 & 0 & 0 & 10 & 10 & 239.13 & 6146 \\
\hline 12 & 217.56 & 1 & 1 & 0 & 0 & 0 & 1 & 1 & 172.62 & 32.54 & 0 & 0 & 0 & 10 & 10 & 225.16 & 5649 \\
\hline 13 & 207.20 & 1 & 1 & 0 & 0 & 0 & 1 & 1 & 163.31 & 30.71 & 0 & 0 & 0 & 10 & 10 & 214.02 & 5263 \\
\hline 14 & 196.84 & 1 & 1 & 0 & 0 & 0 & 1 & 1 & 154.02 & 28.90 & 0 & 0 & 0 & 10 & 10 & 202.92 & 4887 \\
\hline 15 & 227.92 & 1 & 1 & 0 & 0 & 0 & 1 & 1 & 182.66 & 34.51 & 0 & 0 & 0 & 10 & 10 & 237.17 & 6076 \\
\hline 16 & 233.10 & 1 & 1 & 1 & 0 & 0 & 1 & 1 & 185.41 & 35.05 & 1.36 & 0 & 0 & 10 & 10 & 241.82 & 6249 \\
\hline 17 & 220.15 & 1 & 1 & 0 & 0 & 0 & 1 & 1 & 174.96 & 32.99 & 0 & 0 & 0 & 10 & 10 & 227.95 & 5747 \\
\hline 18 & 230.51 & 1 & 1 & 1 & 0 & 0 & 1 & 1 & 184.30 & 34.83 & 0.02 & 0 & 0 & 10 & 10 & 239.15 & 6147 \\
\hline 19 & 243.46 & 1 & 1 & 1 & 0 & 0 & 1 & 1 & 187.66 & 35.48 & 9.10 & 0 & 0 & 10 & 10 & 252.44 & 6657 \\
\hline 20 & 170.94 & 1 & 1 & 0 & 0 & 0 & 0 & 0 & 148.77 & 27.84 & 0 & 0 & 0 & 0 & 0 & 176.61 & 4678 \\
\hline 21 & 150.22 & 1 & 1 & 0 & 0 & 0 & 0 & 0 & 130.31 & 24.28 & 0 & 0 & 0 & 0 & 0 & 154.59 & 3969 \\
\hline 22 & 179.00 & 1 & 1 & 0 & 0 & 0 & 0 & 0 & 155.72 & 29.19 & 0 & 0 & 0 & 0 & 0 & 184.91 & 4954 \\
\hline 23 & 176.30 & 1 & 1 & 0 & 0 & 0 & 0 & 0 & 153.40 & 28.74 & 0 & 0 & 0 & 0 & 0 & 182.14 & 4861 \\
\hline 24 & 173.10 & 1 & 1 & 0 & 0 & 0 & 0 & 0 & 151.09 & 28.29 & 0 & 0 & 0 & 0 & 0 & 179.38 & 4769 \\
\hline
\end{tabular}

To minimize the use of conventional generators here STPP with total capacity of 20MW is integrated in the system for day time. To optimize the fuel cost of conventional committed generators genetic algorithm (GA) is used. As discussed in above genetic algorithm section parameter selection is done as shown in Table 4. The objective function of GA is the fuel cost obtained in case 2 .

The GA is run one time for each hour. In one-hour GA is run for 100 iterations, in each iteration 100 initial population is generated based on UC-ACOPF unit committed result as obtained is case2. In one iteration out of 100 populations minimum price is saves as 'minc' hence in one iteration 100 'minc' price is generated. Table 5 shows the optimized total fuel cost for conventional committed generators for $24 \mathrm{hr}$ load schedule. As shown in table total saving in $24 \mathrm{hr}$. is $334 \$$.

The peak load hour is $19^{\text {th }}$ hour, GA is optimized at $98^{\text {th }}$ iteration with minimum price of $6657(\$)$. Figure 3 show the 'minc' plot obtained for $19^{\text {th }} \mathrm{hr}$. for all three committed generators. Figure 4 show the optimization characteristic of objective function that is total cost for $19^{\text {th }} \mathrm{hr}$. Power generation for each committed generator is shown in Figure 5. 
Table 3. Hourly Min. and Max. Load Bus Voltages for IEEE 14 Bus Test System with STPP

\begin{tabular}{|c|c|c|c|c|}
\hline Time (hour) & $\mathbf{V}_{\max }(\mathbf{p} . \mathbf{u})$ & $\mathbf{V}_{\text {min }}(\mathbf{p . u})$ & $\begin{array}{l}\text { Active loss(P) } \\
\quad \text { (MW) }\end{array}$ & $\begin{array}{l}\text { Reactive } \operatorname{loss}(\mathbf{Q}) \\
\text { (MVAR) }\end{array}$ \\
\hline 1 & 1.060 & 1.024 & 1.42 & 4.35 \\
\hline 2 & 1.060 & 1.032 & 0.69 & 2.11 \\
\hline 3 & 1.060 & 1.033 & 0.63 & 1.91 \\
\hline 4 & 1.060 & 1.033 & 0.63 & 1.91 \\
\hline 5 & 1.060 & 1.029 & 0.98 & 3.00 \\
\hline 6 & 1.060 & 1.019 & 1.95 & 5.96 \\
\hline 7 & 1.060 & 1.019 & 1.95 & 5.96 \\
\hline 8 & 1.060 & 1.016 & 2.44 & 7.45 \\
\hline 9 & 1.060 & 1.015 & 2.26 & 6.89 \\
\hline 10 & 1.060 & 1.013 & 2.64 & 8.07 \\
\hline 11 & 1.060 & 1.012 & 2.71 & 8.27 \\
\hline 12 & 1.060 & 1.015 & 2.38 & 7.27 \\
\hline 13 & 1.060 & 1.030 & 0.86 & 2.64 \\
\hline 14 & 1.060 & 1.018 & 1.90 & 5.81 \\
\hline 15 & 1.060 & 1.013 & 2.60 & 8.12 \\
\hline 16 & 1.060 & 1.012 & 2.74 & 8.36 \\
\hline 17 & 1.060 & 1.014 & 2.45 & 7.47 \\
\hline 18 & 1.060 & 1.015 & 2.80 & 8.55 \\
\hline 19 & 1.060 & 1.015 & 2.86 & 8.72 \\
\hline 20 & 1.060 & 1.025 & 1.73 & 5.28 \\
\hline 21 & 1.060 & 1.025 & 1.33 & 4.06 \\
\hline 22 & 1.060 & 1.020 & 1.90 & 5.79 \\
\hline 23 & 1.060 & 1.020 & 1.84 & 5.62 \\
\hline 24 & 1.060 & 1.021 & 1.78 & 5.45 \\
\hline
\end{tabular}

Table 4. Parameters selection

\begin{tabular}{|c|c|}
\hline Parameters & Specifications \\
\hline Population size & 50 \\
\hline Crossover probability & 0.5 \\
\hline Mutation probability & 0.15 \\
\hline Maximum iteration & 100 \\
\hline
\end{tabular}


Table 5. Optimal result of GA for 24-hour UC schedule

\begin{tabular}{|c|c|c|c|c|c|c|c|c|c|c|c|}
\hline \multirow{2}{*}{$\begin{array}{c}\text { Time } \\
\text { (hr) }\end{array}$} & \multirow{2}{*}{$\begin{array}{l}\text { Total } \\
\text { Power } \\
\text { (MW) }\end{array}$} & \multirow{2}{*}{$\begin{array}{c}\text { No: of } \\
\text { iterations }\end{array}$} & \multicolumn{7}{|c|}{ Power (in MW) } & \multirow{2}{*}{$\begin{array}{c}\text { Total } \\
\operatorname{cost}(\$ / h r) \\
\text { (with GA) }\end{array}$} & \multirow{2}{*}{$\begin{array}{c}\text { Total Cost } \\
\text { (\$/hr) }\end{array}$} \\
\hline & & & $P g_{1}$ & $P \boldsymbol{g}_{2}$ & $\boldsymbol{P g}_{3}$ & $\mathrm{Pg}_{4}$ & $\boldsymbol{P g}_{5}$ & $\boldsymbol{P} \boldsymbol{g}_{13}$ & $\boldsymbol{P g}_{14}$ & & \\
\hline 1 & 160.00 & 97 & 134 & 26 & 0 & 0 & 0 & 0 & 0 & 4135 & 4142 \\
\hline 2 & 111.00 & 96 & 94 & 17 & 0 & 0 & 0 & 0 & 0 & 2659 & 2675 \\
\hline 3 & 106.00 & 97 & 89 & 17 & 0 & 0 & 0 & 0 & 0 & 2518 & 2524 \\
\hline 4 & 106.00 & 92 & 89 & 17 & 0 & 0 & 0 & 0 & 0 & 2512 & 2524 \\
\hline 5 & 133.00 & 99 & 112 & 21 & 0 & 0 & 0 & 0 & 0 & 3294 & 3302 \\
\hline 6 & 188.00 & 98 & 158 & 30 & 0 & 0 & 0 & 0 & 0 & 5030 & 5047 \\
\hline 7 & 188.00 & 97 & 157 & 31 & 0 & 0 & 0 & 0 & 0 & 5022 & 5047 \\
\hline 8 & 210.00 & 95 & 176 & 34 & 0 & 0 & 0 & 0 & 0 & 5808 & 5819 \\
\hline 9 & 220.00 & 99 & 168 & 32 & 0 & 0 & 0 & 10 & 10 & 5438 & 5455 \\
\hline 10 & 236.00 & 97 & 182 & 34 & 0 & 0 & 0 & 10 & 10 & 6021 & 6046 \\
\hline 11 & 239.00 & 97 & 184 & 35 & 0 & 0 & 0 & 10 & 10 & 6124 & 6146 \\
\hline 12 & 225.00 & 96 & 173 & 32 & 0 & 0 & 0 & 10 & 10 & 5646 & 5649 \\
\hline 13 & 214.00 & 98 & 163 & 31 & 0 & 0 & 0 & 10 & 10 & 5247 & 5263 \\
\hline 14 & 203.00 & 97 & 154 & 29 & 0 & 0 & 0 & 10 & 10 & 4875 & 4887 \\
\hline 15 & 237.00 & 98 & 183 & 34 & 0 & 0 & 0 & 10 & 10 & 6054 & 6076 \\
\hline 16 & 242.00 & 99 & 184 & 36 & 2 & 0 & 0 & 10 & 10 & 6244 & 6249 \\
\hline 17 & 228.00 & 99 & 175 & 33 & 0 & 0 & 0 & 10 & 10 & 5739 & 5747 \\
\hline 18 & 239.00 & 98 & 183 & 36 & 0 & 0 & 0 & 10 & 10 & 6136 & 6147 \\
\hline 19 & 253.00 & 98 & 188 & 36 & 9 & 0 & 0 & 10 & 10 & 6654 & 6657 \\
\hline 20 & 177.00 & 97 & 149 & 28 & 0 & 0 & 0 & 0 & 0 & 4641 & 4678 \\
\hline 21 & 155.00 & 99 & 130 & 25 & 0 & 0 & 0 & 0 & 0 & 3967 & 3969 \\
\hline 22 & 185.00 & 98 & 156 & 29 & 0 & 0 & 0 & 0 & 0 & 4941 & 4954 \\
\hline 23 & 182.00 & 93 & 153 & 29 & 0 & 0 & 0 & 0 & 0 & 4846 & 4861 \\
\hline 24 & 179.00 & 90 & 151 & 28 & 0 & 0 & 0 & 0 & 0 & 4749 & 4769 \\
\hline \multicolumn{10}{|c|}{ Overall cost } & 1,18,299.0 & $1,18,633.0$ \\
\hline
\end{tabular}


Figure 3. Minimum cost function (minc) plot

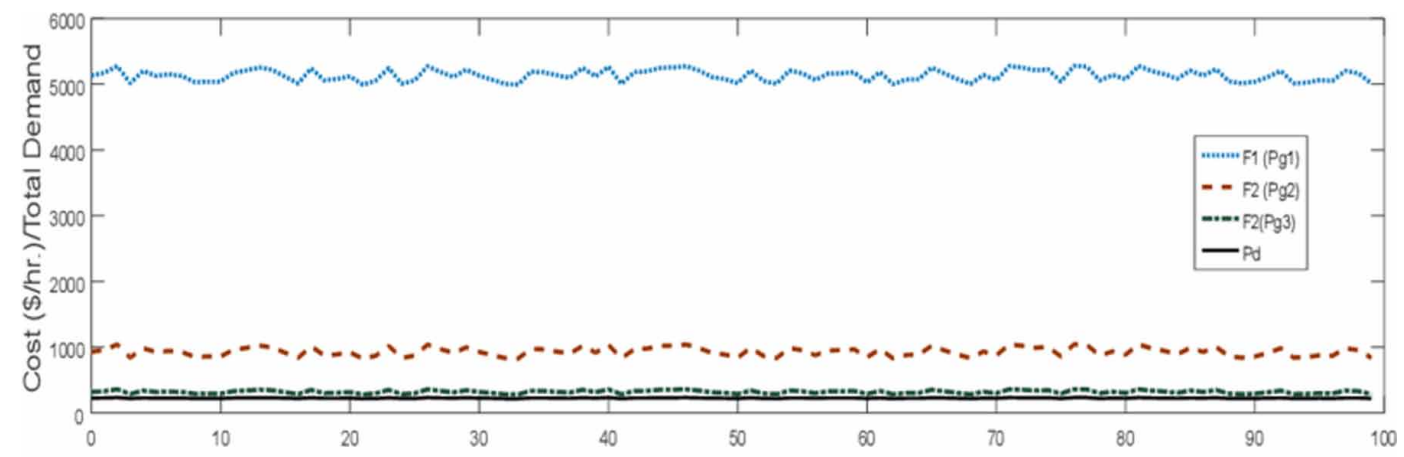

No. of iterations

Figure 4. Objective function optimization characteristic

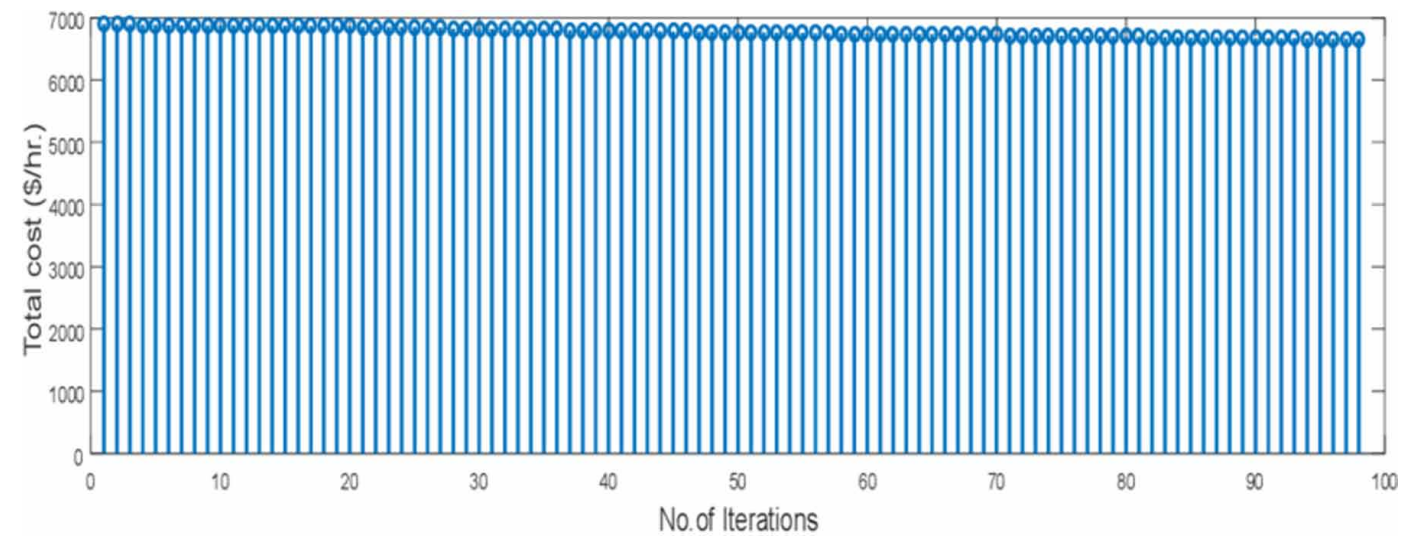

Figure 5. Power Generation plot

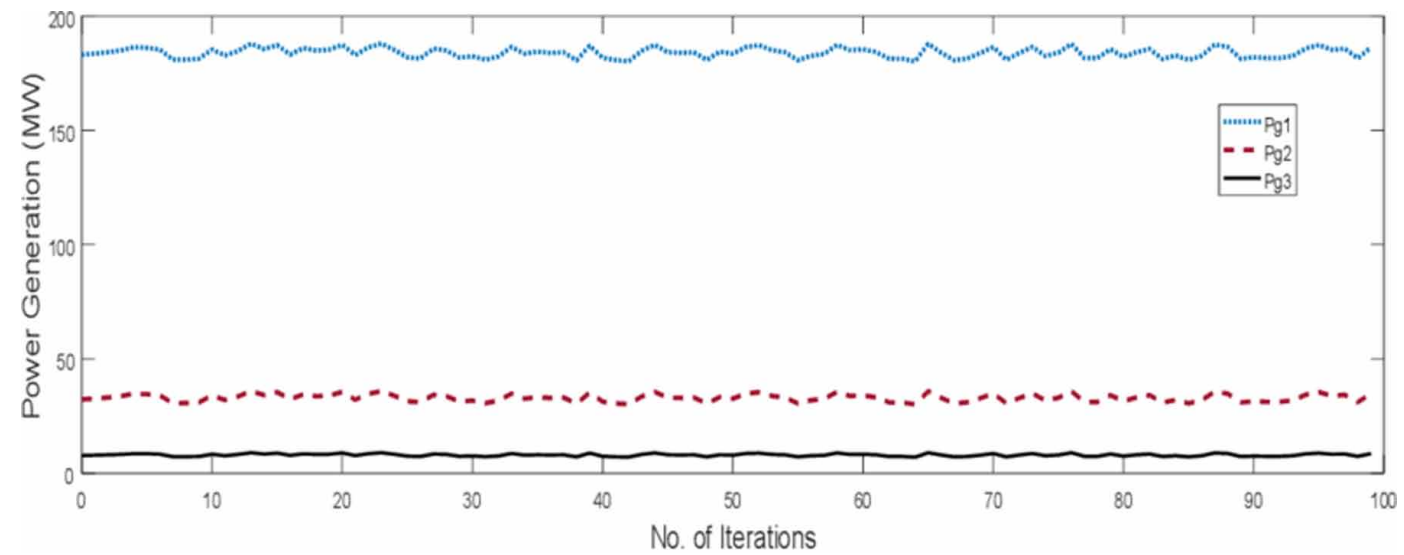




\section{CONCLUSION}

Unit Commitment is a complex problem of optimization in power systems. A prior knowledge of the units to be committed among the available to meet the anticipated load demand not only reduces the generation expense, but also allows system operators to operate smoothly. Many approaches can solve the unit commitment problem to get the optimal solution. The question of unit commitment is nothing; it's the basic load study expected at different time schedules. In this paper, one day (24 hour) load profile is used to show the effectiveness of the proposed approach. The STPP installation method is also proposed effectively on the selected system. A genetic algorithm-based approach has been successfully proposed and applied to minimize the fuel cost of conventional unit committed generators. The proposed approach compares the fuel cost with and without GA and show the saving of fuel cost by using GA. 


\section{REFERENCES}

Baporikar, N. (2020). Logistics Effectiveness Through Systems Thinking. International Journal of System Dynamics Applications, 9(2), 1-16.

Bhardwaj, A., Kamboj, K., Shukla, V., Singh, B., \& Khurana, P. (2012). Unit commitment in electrical power system-A literature review. IEEE International Power Engineering and Optimization Conference Melaka, 275-280.

Carrion, M., \& Arroyo, J. (2011). A computationally efficient mixed-integer linear formulation for the thermal unit commitment problem. IEEE Transactions on Power Systems, 69-77.

Columbus, C. C., \& Simon, S. P. (2012) Hybrid Particle swarm approach for Security constrained unit commitment. International Conference on Computing, Electronics and Electrical Technologies (ICCEET), 128-133.

Danwen, Y. U., Ming, Y., \& Zhai, H. (2016). An overview of robust optimization used for power system dispatch and decision-making. Dianli Xitong Zidonghua, 40(7), 134-143.

Feizollahi, M. J., Costley, M., Ahmed, S., \& Grijalva, S. (2015). Large-scale decentralized unit commitment. International Journal of Electrical Power \& Energy Systems, 73, 97-106. doi:10.1016/j.ijepes.2015.04.009

Galli, B. J. (2019). Thoughts of Using Economic Decision-Making to Systems Engineering and Systems Thinking: An Exploratory Study. International Journal of System Dynamics Applications, 8(3), 1-14.

Garver, L. L. (1962). Power generation scheduling by integer programming-Development and theory. Trans. Amer. Inst. Elect. Eng. Power App Syst, 730-734.

Gupta, N. K., Singh, A. K., Thombre, A. D., \& Pal, K. (2018). Smart Solar Energy Management to Power Computer Lab in Rural Areas. In 3rd International Innovative Applications of Computational Intelligence on Power, Energy and Controls with their Impact on Humanity. CIPECH.

Hirpara, H. R., \& Sharma, S. N. (2020). An Analysis of a Wind Turbine-Generator System in the Presence of Stochasticity and Fokker-Planck Equations. International Journal of System Dynamics Applications, 9(1), 1-26.

Jabr, R. A. (2012). Tight polyhedral approximation for mixed-integer linear programming unit commitment formulations. IET Generation, Transmission \& Distribution, 1104-1111.

Kumar, D., Kumar, A., \& Yadav, L. K. (2014). Unit commitment of thermal power plant in integration with wind and solar plant using genetic algorithm. International Journal of Engineering Research \& Technology (Ahmedabad), 3(7), 664-669.

Lambora, A., Gupta, K., \& Chopra, K. (2019). Genetic Algorithm- A Literature Review. International Conference on Machine Learning, Big Data, Cloud and Parallel Computing (COMITCon), 380-384.

Meshram, U., Morey, S., \& Gawande, S. (2016). To Study and Analyze Economic Load Dispatch in Power System Using Genetic Algorithm. International Journal of Innovative Research in Electrical, Electronics, Instrumentation and Control Engineering, 4(4), 231-235.

Mohamed, G. R., Ebrahim, M. A., Bendary, F. M., \& Osman, S. A. A. (2017). Transient Stability Enhancement for 20 MW PV Power Plant via Incremental Conductance Controller. International Journal Of System Dynamics Applications, 6(4), 1-22.

Morales, J. M., Conejo, A. J., \& Pérez-Ruiz, J. (2009). Economic valuation of reserves in power systems with high penetration of wind power. IEEE Transactions on Power Systems, 900-910.

Morales-Espana, G., Latorre, J. M., \& Ramos, A. (2013). Tight and compact MILP formulation for the thermal unit commitment problem. IEEE Transactions on Power Systems, 4897-4908.

Ostrowski, J., Anjos, M. F., \& Vannelli, A. (2012). Tight mixed integer linear programming formulations for the unit commitment problem. IEEE Transactions on Power Systems, 39-46.

Padhy, N. P. (2004). Unit commitment-A bibliographical survey. IEEE Transactions on Power Systems, 19(2), 1196-1205. doi:10.1109/TPWRS.2003.821611 
Parashar, A., \& Swankar, K. K. (2013). A Genetic Algorithm Approach to Solve Unit Commitment Problem. Journal of Electrical and Electronics Engineering (Oradea), 7(3), 60-64.

Ramadan, R. A., \& Altamimi, A. B. (2017). Hierarchal Fuzzy Logic Controller and Internet ofThings (IoT) Information: Disease Spreading as a Test Case. International Journal of System Dynamics Applications, 6(3), $1-28$.

Salima, M., Loubna, A., \& Riad, T. (2018). A Global Stability of Linearizing Control of Induction Motor for PV Water Pumping Application. International Journal of System Dynamics Applications, 7(3), 1-26.

Shin, S. S., Oh, J. S., \& Jang, S. H. (2017). Active and reactive power control of ESS in distribution system for improvement of power smoothing control. Journal of Electrical Engineering \& Technology, 12(3), 1007-1015.

Singh, A. K., Pal, K., \& Gupta, N. K. (2019). Performance Evaluation of a 4 kW Isolated Solar Powered Lab with IoT Energy Management System. 2nd International Conference on Power Energy. Environment and Intelligent Control (PEEIC), 418-423.

Stott, B., \& Alsac, O. (2012). Optimal power flow: Basic requirements for real-life problems and their solutions. Academic Press.

Wei, C., Xin, A. I., \& Tao, W. U. (2013). Influence of grid-connected photovoltaic system on power network. Dianl Zidonghua Shebei, 33(2), 26-32, 39.

Wu, L., \& Shahidehpour, M. (2010). Accelerating Benders decomposition for network-constrained unit commitment problems. Energy Syst., 1(3), 339-376. doi:10.1007/s12667-010-0015-4

Xua, J., \& Pei, A. (2018). Application of Genetic Algorithm with Random Crossover and Dynamic Mutation on the Travelling Salesman Problem. 8th International Congress of Information and Communication Technology (ICICT). Procedia Computer Science, 131, 937-945.

Yatimi, H., \& Aroudam, E. (2018). Standalone Photovoltaic System with Maximum Power Point Tracking: Modeling and Simulation. International Journal of System Dynamics Applications, 7(3), 1-18.

Zhao, B., Guo, C. X., Bai, B. R., \& Cao, Y. J. (2006). An improved particle swarm optimization algorithm for unit commitment. International Journal of Electrical Power \& Energy Systems, 28(7), 482-490. doi:10.1016/j. ijepes.2006.02.011

Aniket Agarwal has completed his integrated dual degree in B.Tech(Electrical Engineering) and M.Tech(Power System Engineering) from electrical branch in 2020 from Gautam Buddha University, School of Engineering, Greater Noida, India.

Kirti Pal is an Associate Professor in Electrical Engineering Department, School of Engineering, Greater Noida, India. She has total 14 year of teaching experience. Dr. Kirti holds a PhD degree in Electrical Engineering, 2013 from RGTU, Bhopal; M.E. degree from MITS Gwalior, 2006; BE degree from L.N.C.T. Bhopal, 2004. She has published many research and conference paper in various reputed journals and conferences. She has guided project and dissertation work of many B.Tech and M.tech Students. She has delivered many keynote and expert talks in national/international conferences/workshop. Her research areas are Restructuring of Power System, Power System Analysis and optimization, Soft Computing Techniques, Renewable Energy systems and Electric Vehicle. 